\title{
Evaluation on the Agricultural Website's Efficiency Based on DEA Method
}

\author{
Shangmin Deng ${ }^{1}$ and Weili Men ${ }^{2}$ \\ ${ }^{1}$ Headmaster's Office of Shandong \\ University of Technology, Zibo, P.R.China \\ deng2132@sdut. edu.cn \\ ${ }^{2}$ Science and Technology Information Research Institute \\ of Shandong University of Technology \\ 346380987@163.com
}

\begin{abstract}
An information-driven agriculture is firstly agriculture digitalization, the foundation of which is the construction of agricultural information networks. Based on the methods of website evaluation, an indicator system of efficiency evaluation for agricultural information websites is constructed in this thesis. Then the efficiency of 17 municipal websites in Shandong province are analyzed by the C2R Model in DEA. The result declares that 3 of these websites are related effective and the other 14 are non-effective. By an analysis of projection, this thesis also gives suggestions to improve the performance of the non-effective.
\end{abstract}

Keywords: Agricultural information website, Efficiency of website, Data Envelopment Analysis (DEA), Evaluation research.

\section{Introduction}

Issues concerning agriculture, countryside and farmers, are called Three Rural Questions, which take an crucial part in the realization of the goal of a well-to-do society and the development of our country's economy. It is acknowledged that it will be harder for uninformed districts to develop their own agriculture. Nowadays, the information gap between urban and rural areas has attracted wide attention of experts from Information Science. The Party Central Committee, since 16th party congress ,hammers away at the point that we should always take addressing the problems facing China's agriculture as the top priority of our economic, and also put forward "to boost the county economy" at the same time. One of "the Three Most Requirement", raised on the Third Plenary Session of the 11th Central Committee of the C.P.C, is that the position of agriculture as the foundation is still weak and need to brush up on the most. In March 2010,the government of Shandong province makes the implementation of the plan named "Powerful Province of information" and the development of Digital Agriculture positively as key points to promote new Information Industries.[1] 
The informationization of agriculture, in the first place, is digitalization, which means to devote major efforts to develop the digital agriculture. As we all know that, the foundation of digitalization is the construction of information networks and the service level is determined by such factors as the resources ,the infrastructural facilities and the environment of informationization, as well as the human resources and the effectiveness of agricultural information services .In another word ,the informationization of agricultural service can be demonstrated by that of agriculture.[2] So, it is reasonable to estimate the present situation of agriculture services .However, because of the difference in geographical conditions and culture backgrounds, we need a appropriate method to selected samples comprehensively and objectively. Fortunately there is a successful precedent created by the DEA (Data Envelopment Analysis for short) which is convenient and practical.[3]

\section{Data Envelopment Analysis}

DEA which is short for Data Envelopment Analysis is a new system analytic method.It was firstly put forward by two operational research experts, A.Charnes and W.W.Copper, to deal with inputs and outputs subject to random disturbances, according to the relative efficiency and evaluation of returns for familiar departments. Based on the concept of relative efficiencies, this method evaluates the relative efficiency of DMUs (Decision Making Units for short) by linear programming model. Besides evaluate and list DMUs as relative efficiency, the results also will point out existing problems and improvement for non-efficient DMUs. Furthermore, instead of setting the weight of inputs/outputs beforehand, DEA gets objective value for each random variable by computing actual datas, which makes the conclusion independently and impartially.[4]

In the light of different research purposes, there are many different DEA models. In this thesis, we select the C2R model just because we want to study whether the DMUs are technique and scale simultaneously efficient when to decrease its inputs by suitable times with its unchanged outputs. The DMU is technique or scale efficient unless it is DEA efficient; otherwise, it will be not efficient.

\section{Description of the Model of $C^{2} R$}

As the first evaluation model, the results of $C^{2} R$ can be used to demonstrate the efficient of DMUs. For the sake of our discussion we assume that the number of DMUs is $\mathrm{n}$, and the number of input and output variables each DUM has is " $\mathrm{m}$ " and "s" respectively. we also set up a mathematical formula of the relationship for the two kinds of variables to fulfill:

$X j=(X 1 j, X 2 j, X 3 j, \ldots, X m j) T \geq 0$,

$Y j=(Y 1 j, \quad Y 2 j, \quad Y 3 j, \quad \ldots, \quad Y s j) T \geq 0, j=1, \ldots, n$;

At the same time, other certain conditions should be satisfied:

$\mathrm{Xij}>0, \mathrm{Yrj}>0, \mathrm{i}=1,2, \ldots, \mathrm{m} ; \mathrm{r}=1,2, \ldots, \mathrm{s}$. 


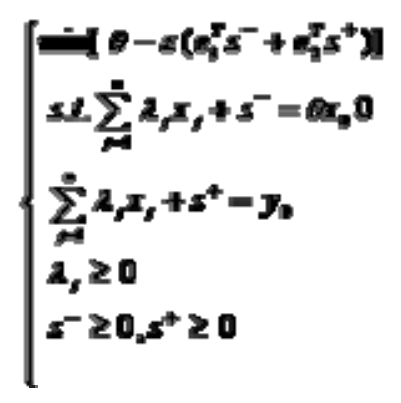

The model is known as efficiency evaluation of decision-making units such as j0, subject to meeting the efficiency of all the DUMs at checkpoints, then we come to a $\mathrm{C}^{2} \mathrm{R}$ modle with non archimedean infinitesimal vector. The optimality is relative efficiency of a DMU, as contrasted with other DUMs, according to what have we suggested. [5]

Here, we use $\theta$ as the input proportional variable, $\varepsilon$ as the non archimedean infinitesimal vector (usually, we let it be $10-6) ; \boldsymbol{G}_{\mathbf{T}}^{\mathbf{T}}=(1,1 \ldots, 1) \in \mathrm{Em}, \mathbf{S}_{\mathbf{T}}^{\mathbf{T}}=(1$, 1..., 1) $\in$ Es; $\boldsymbol{s}^{-}=\left(\boldsymbol{s}_{\mathbf{1}}^{-}, \boldsymbol{s}_{\mathbf{2}}^{-}, \ldots \boldsymbol{s}_{\mathbf{n}}^{-}\right)$is slack variables correspond to inputs, $\boldsymbol{s}^{+}=$

$\left(\mathbf{s}_{\mathbf{1}}^{\mathbf{*}} \mathbf{x}_{\mathbf{2}}^{\mathbf{+}} \ldots \mathbf{s}_{\mathbf{s}}^{\mathbf{*}}\right)$ is the variable to output and the $\lambda \mathrm{j}$ is the decision variable for the appointed DMU.

When this model is applied to data, we will get the optimal solution composed of $\lambda \mathrm{j}, \quad \mathrm{S}^{*_{-}}, \mathrm{S}^{*}+$ and $\theta^{*}$.There are several results that we should make into our consideration:

(1) When $\theta 0=1$, s0- $=0$ and $s 0+=0$, we think the DMU is efficiency which means technique and scale are simultaneously efficient.

(2) When $\theta 0=1$, - but $s 0-\neq 0$ or $s 0+\neq 0$, we believe that under some conditions the DMU is weak DEA efficient. In other words, the either technique or scale is efficient.

(3) When $\theta 0<1$, the performance of this DUM is of non-effective.

Therefore, we consider it as Effective Decision-making Units when $\theta 0=1$; otherwise, it is ineffective. [6]

\section{Efficiency Evaluation on Agriculture Websites}

Now, the number of municipal cities in Shandong province is about 91, which counts for $65 \%$. Intra-county economy plays a vital role in the development in the informationalization of our province. Even though, much attention has been paid on the informationization of agriculture, there are still many problems that should take into our focus. Researches on agriculture websites will not only improve the design and maintenance for websites of corporations, but also promote the standardization of the management and speed up the process of agricultural modernization in Shandong Province. [7] 


\subsection{Evaluating Index}

The purpose of this thesis is to evaluate the agriculture websites in Shandong province. The main method is to study the inputs effectiveness while keep outputs steady. To make the result objective and easy to analyze, we choose such inputs (inps for short) variables as TAG (Total number of web pages), APS (Average page size), TFS (Total foreign sites that link to this site), UDR (Updata rating), NBL (No broken links), FRP (Foreign (external) pages referenced), NRP (Non returning percentage) and $\mathrm{CP}$ (Connected percentage).[8]

TAG is the number of static and dynamic pages belonging to the same root directory. APS describes the average pages size classified by 10k times.TFS evaluates the networks, inputs variables effectiveness from the perspective of website promotion. UDR is a relative concept. Maintainers usually update different content types in different times. So before we estimate the upiea objectively, we need to classified different updating periods in sorts. In this thesis, we divide the periods into four catalogues. According to the different influence, we give weights to the periods respectively. NBL means using this kind of links, we can not find the target website. Though this, we have another way to measure the effective of websites. The more FRP is, the longer time it will take to operate the website. NRP have the similar function as CP. Both of them evaluate the effectiveness of website from the eye of site navigations.

Output (outp for short) variables we choose means the number of visitors to the site, which has a certain relation with TFS. Because a visitor may glance over many different documents or more than one websites, the clicks may be much larger. [9]

\subsection{Sample}

To determine the Agricultural Website's Efficiency, we choose 17 sites as DMUs set, built by Agricultural Information Centre of Shandong province and subordinate departments. They have common in many aspects such as contents, receivers and functions, which meet the requirements for application of $C^{2} R$ model in DEA.

\subsection{Data}

Using Maxamine[10], a specialized profiler to make a analysis of networks, the mentioned sites were scanned. The objective datas we collected includes the internal information of the sites and interconnection information between networks, among which, the UDR are calculated as table 1.

The output consult verified test results from alexa.com, an authoritative Thirdparty certification website, applying the number of visitor for the targeted site.

Table 1. Calculation methods of update rate of website

\begin{tabular}{llll}
\hline update cycle of pages & update rate Ri & Weight $\lambda$ & update rate of website \\
\hline$\leq 1$ week & R1 & 0.50 & \\
1 week $\sim 6$ weeks & R2 & 0.30 & $\boldsymbol{U D R}=\mathbf{R} * \mathbf{A}$ \\
6 weeks $\sim 6$ months & R3 & 0.15 & \\
$\geq 6$ months & R4 & 0.05 & \\
\hline
\end{tabular}


Table 2. Appraisal index of the efficiency of websites in shandong province

\begin{tabular}{cccc}
\hline $\begin{array}{c}\text { name of } \\
\text { counties }\end{array}$ & $\begin{array}{c}\text { the efficiency } \\
\text { of website }\end{array}$ & $\begin{array}{c}\text { name of } \\
\text { counties }\end{array}$ & $\begin{array}{c}\text { the efficiency } \\
\text { of website }\end{array}$ \\
\hline Qingdao & 1.000 & Dongying & 0.284 \\
Weihai & 1.000 & Weifang & 0.243 \\
Jinan & 1.000 & Binzhou & 0.143 \\
Zibo & 0.662 & Linyi & 0.122 \\
Liaocheng & 0.494 & Dezhou & 0.057 \\
Laiwu & 0.439 & Tai-an & 0.055 \\
Jining & 0.395 & Zaozhuang & 0.050 \\
Yantai & 0.362 & Rizhao & 0.042 \\
Heze & 0.315 & & \\
\hline
\end{tabular}

Form table 2, we can see that three DUMS are of effective units in DEA. In other words, the others 14 are non DEA efficient, among which, there are four websites have an effectiveness below 0.01. Therefore, it can be concluded that, the agricultural sites, efficiency in Shandong are lower than expected. Then, we make an projection analysis for the non DEA efficient based on the DEAP2.1 results. The main task is to analyze relative improvement of inputs when outputs is remain unchanged. We also calculate potential improvements of inputs and outputs under ideal condition.

The average potential improvements are showed in table 3.The potential improvements demonstrate that, reference to the non DEA efficient DMUs, we have not make full use of each input already, so, in theory at least, we can promote the sites efficiency and induce inputs. If we keep inputs still, the output is also can be promoted by $61 \%$. In other words, if inputs can not be reduced, theoretical, efficiency can be raised by the increasing number of visitors.

Table 4 shows main factors influencing websites efficiency, including FRP, TAG, NRP and APS.

\subsection{Recommendations}

From table 2, we know that the operation efficiencies are very different among these 17 municipal agriculture websites, even though most of them are inefficient. Reference to non DEA efficient, combined with the analysis of table 3 and table 4 , we suggest that the relative departments should take such actions as following to improve the performance of the non DEA efficient:

Table 3. The mean potential improvements for input and output indexes

\begin{tabular}{lccccccccc}
\hline item & Inp 1 & Inp 2 & Inp 3 & Inp 4 & Inp 5 & Inp 6 & Inp 7 & Inp8 & Outp \\
\cline { 2 - 8 } & TAG & APS & TFS & UDR & NBL & FPR & NRP & CP & Reach \\
\hline $\begin{array}{l}\text { mean } \\
(\%)\end{array}$ & -61 & -46 & -46 & -50 & -58 & -75 & -55 & -61 & 61 \\
\hline
\end{tabular}


Table 4. Analysis on the main influence factors for inefficient DMUs

\begin{tabular}{llll}
\hline \multicolumn{1}{c}{ website } & Compared Item & \multicolumn{1}{c}{ website } & Compared Item \\
\hline www.zbny.gov.cn & APS & www.wfny.gov.cn & TFS,FPR \\
www.lcagr.cn & TAG,FPR,NRP & www.bzny.gov.cn & TFS,NRP \\
agri.laiwu.gov.cn & TAG,APS,NBL, & $\underline{\text { www.lyny.gov.cn }}$ & TAG \\
www.kelawang.com & TAG,FPR & www.dzny.gov.cn & TAG,NBL,NRP \\
www.ytny.gov.cn & TAG,UDR,NBL, & www.tany.gov.cn & TFS,FPR,NRP \\
www.hzsny.gov.cn & TAG,APS,UDR, & www.zznjh.gov.cn & FPR,NRP \\
www.dyny.gov.cn & FPR & www.rzny.gov.cn & APS,FPR,NRP \\
\hline
\end{tabular}

\begin{abstract}
The target of this action is to reduce TAG and lower APS. The two aspects not only reflect the information website contents, but also the website's usability and security. Agriculture sites are made for agriculture participants and relative departments. So early in the website construction, we need to consider how to design the pages reasonably, and abstract the content to ensure its usability and security.
\end{abstract}

Cut down NRP. NRP evaluates websites from the perspective of navigation. This aspect means reducing inputs of navigation. If the site has a set Robustness, the more complexity the navigation is, the easier visitors will be lost. This, finally, will indirectly affect the website's efficiency. If the navigation is simplified, not only the usability is improved, but also the inputs of the operation can be cut down.

Reduce TFS. This method includes reducing inputs of website promotion or increase the number of the visitors. On some degree, TFS represents the stability of a website, yet the more external links are, the higher it will cost. Checking dead links, updating old links and other resources maintenance will affect the operation efficiency. So, in order to promote the efficiency, we need to reduce TFS while the quality of the web is ensured. Only in this way, can we attract more attention and keep the site authority.

\title{
5 Conclusions
}

Though all the above mentioned, we have a clear idea that there are 14 websites are non DEA efficient, which counts for $84.3 \%$ of all municipal websites in Shandong province. We have presented a detailed accounting of efficiency of agricultural websites and suggestions are also given to improve the current situation. Evaluation of the agriculture websites' efficiency can not only reflect the present situation of Shandong agriculture, but also be valuable for further development in the construction of information agriculture and application of related net resources. 


\section{References}

1. Liu, S.H.: Study on the Indicator System for Measuring the Rural Area Informatization Level in China. J. Library and Information Service, 33-36 (1981)

2. Han, X.S., Pan, H.F., Wen, J.H., et al.: Evaluation and Study on Development of Agriculture Information Service. J. Journal of Agricultural Mechanization Research, 20-23 (2007)

3. Hu, P., Fu, Y.Y., Gan, L.: Evaluation on agriculture sites in western area Based on DEA and Cluster analysis. J. Science and Management, 20-22 (2005)

4. Du, D., Pang, Q.H.: Contemporary Comprehensive evaluation methods and classic documentation. Tsinghua University Press, Beijing (2005)

5. Lan, F.: The Evaluation of China s Circulation Economy Efficiency Based on DEA. J. Journal of Harbin University of Commerce (Social Science Edition), 16-19 (2000)

6. $\mathrm{Wu}, \mathrm{L} .:$ Evaluation on the efficiency of eco-technology Innovation based on DEA. J. Technology Progress and Policy, 114-117 (2009)

7. Tan, L., Wang, X.C.: Study on the Evaluation of Agriculture Web Site. Journal of Anhui Agricultural Sciences, 1876-1882 (2007)

8. Sun, Y.L., He, Y., Zhao, Z.N.: Evaluation on the Western Agricultural Website s Efficiency Based on DEA Method. Journal of Intelligence, 14-17 (2009)

9. Qiu, H.: Theory Research and Application for DEA in websites evaluation. Cambridge University Press, New York (2005)

10. Web Analyst Software Package, http: / / www . maxamine.com 